Estimado compañero:

Habiendo leído el artículo de Francisco Javier Moreno y Sara Cañamón, en el volumen 10, $\mathrm{n}^{\circ}$ 3, pp. 205-219 de 2005 de la revista que diriges, y comoquiera que considero que las alusiones que se hacen en él, tanto las laudatorias - sin sentido alguno si no se justifican y explican-, como aquellas en las que se alude criticamente - e insuficientemente - a la batería EMSDA (ver las escasas nueve líneas de la p. 209 y otras tantas en la p. 216), no se corresponden en absoluto con lo que supone dicho trabajo, acogiéndome al derecho de réplica, me gustaría se publicase la información que adjunto sobre dicha batería, que, a pesar de todas sus insuficiencias, merece ser citada de alguna manera como pionera en el campo de la evaluación de la memoria semántica en español, pero de una manera argumentada y precisa.

Como está publicada tanto en dicho idioma (Peraita et al. 2000) como en inglés (Peraita et al. 2001), no es necesario volver a repetir lo que cualquier lector puede encontrar alli, sino sencillamente profundizar en algunos aspectos de las pruebas que la componen y de los conjuntos de items que las configuran, así como citar algunos de los trabajos originales en los que se ha basado. Además, como se me cita de manera inespecifica en varios lugares, quiero aclarar que no me hago en manera alguna corresponsable de una serie de aspectos del artículo de F.J. Moreno y S. Cañamón (2005) que, a mi modo de ver suponen una serie de errores conceptuales, imprecisiones, lagunas y problemas de diversos tipos.

Le queda agradecida por anticipado en el caso de que decidan publicar esta carta y el documento anejo a la misma

Herminia Peraita Adrados

Catedrática de Psicología Básica de la UNED

\title{
Batería de evaluación de la memoria semántica en demencia tipo Alzheimer (EMSDA; Peraita, González-Labra, Sánchez Bernardos y Galeote, 2000 y 2001): algunos aspectos aclaratorios y complementarios
}

\section{INTRODUCCIÓN}

La batería EMSDA (Evaluación de la Memoria Semántica en la Demencia tipo Alzheimer) está basada, desde el punto de vista teórico, en algunos de los principios subyacentes a la Psicología de Conceptos y Categorías que rigieron este ámbito de la Psicología Cognitiva en los años 70-90, a partir, sobre todo, de las investigaciones de Eleanor Rosch y la escuela que en cierta manera surgió de ella (Rosch, 1976; Smith y Medin, 1981). En consecuencia, las predicciones e hipótesis de la Batería parten de estos mismos presupuestos conceptuales. Por ello, además de una selección equilibrada de los items entre las categorias de seres vivos y seres no vivos (de ahora en adelante SVs y SNVs) ${ }^{1}$, se par- te siempre que es posible de distintos niveles de generalidad de los items - supraordenado y básico- y se controla la tipicidad de los mismos a partir de las únicas normas existentes en español cuando se diseñó la batería (Soto et al., 1994).

Los análisis estadisticos y psicometricos a los que se sometio la Bateria EMSDA demostraron la validez de constructo y valor predictivo de la misma para detectar deterioro cognitivo en la memoria semántica en EA leves y moderados, así como en pacientes con posible demencia semántica y otros problemas del sistema semántico (EHS: encefalitis por herpes simple, TCE: traumatismo cráneo-encefalico, etc.).

IO categorias naturales y de artefactos en terminologia de la Epoca. 
Tabla 1. Clasificación de las pruebas en función del tipo de procesamiento implicado y de la modalidad sensorial: perceptivo- visual y verbal-auditiva (ya que todas las pruebas linguísticas fueron presentadas oralmente)

\begin{tabular}{lcc}
\hline & Visual & Verbal \\
\hline Producción & $\begin{array}{c}\text { Denominación } \\
\text { Clasificación }\end{array}$ & Fluidez \\
& Eefinición de categorías \\
\hline Comprensión & $\begin{array}{c}\text { Emparejamiento } \\
\text { Analogías Verificación }\end{array}$ & Reconocimiento atributos \\
\hline
\end{tabular}

En la actualidad, y tras varios años de intensos debates sobre la naturaleza, entidad, principios de organización y representación de la memoria semántica (llevados a cabo sobre todo desde el ámbito de la neuropsicología cognitiva), vuelve a renacer el interés por algunos de los pilares básicos sobre los que se construyó aquella Psicología sobre las Categorías y los Conceptos $\mathrm{y}$, además, para integrar datos a menudo contradictorios - por ejemplo, los procedentes de los innumerables estudios empíricos sobre las disociaciones categoriales, se detecta la necesidad de replantearse los hallazgos, fenómenos experimentales y mecanismos explicativos en que se basaba buena parte de la literatura científica de aquella época.

Volviendo a la batería EMSDA, esta consta de ocho pruebas, que se articulan en torno a los dos grandes procesos cognitivo-lingüísticos: producción y comprensión, y en torno a dos modalidades sensoriales de presentación de los items: perceptivovisual y verbal-auditiva, como indica la Tabla 1 .

Algunas de estas pruebas se diseñaron para que fueran complementarias entre sí y, por eso, se relacionan dos a dos: por ejemplo, las pruebas de Definición de categorias y Reconocimiento de atributos, o las de Fluidez Verbal y Clasificación. El objetivo es siempre tratar de obtener una convergencia de indicios en los resultados.

Se cita la fuente y /o el marco teórico en el que se inspira cada una de las ocho pruebas, así como el objetivo o finalidad en términos de procesamiento de la información semántica, también en cada una de ellas, y se explica el minucioso sistema de puntuación adoptado, diferente para cada una de las pruebas y que va más allá del mero cómputo de aciertos, errores y omisiones, que es habitual en la mayoría de las pruebas o tests al uso en este ámbito.

Si bien en la primera prueba, Fluidez Verbal, se parte de cinco categorias semánticas (en general los trabajos de este tipo parten tan sólo de una o dos categorias) de nivel supraordenado (ver Tabla 1), categorías que se repiten a lo largo de todas las pruebas, el número total de categorias usadas a lo largo de las ocho pruebas de que consta la Batería, es de 176 , más o menos el $50 \%$ presentadas en formato lingüístico y el otro $50 \%$ en formato pictórico, consistentes estas últimas en dibujos en blanco y negro diseñados específicamente para esta Batería, aunque en la línea de Snodgrass y Vanderwart (1980).

Por tanto, en su diseño se han combinado los siguientes factores: dos tipos de categorías o dominios semánticos (SVs y SNVs), tipicidad $y / o$ frecuencia de los items, niveles de generalidad (básico y supraordenado) y cuatro tipos de relaciones semánticas ${ }^{2}$ (funcional, parte-todo, perceptual y taxonómica).

1. Dominios:
A. Seres vivos
B. Seres no-vivos

2. Subdominios:
A. Animal
B. Planta
C. Fruta
D. Ropa
E. Vehículo
F. Mueble

3. Valores de tipicidad:
A. Alta
B. Baja

4. Niveles de generalidad:
A. Básico
B. Supraordenado

\footnotetext{
${ }^{2}$ Aunque el número de relaciones semánticas o atributos es mayor que éste en el modelo conceptual de que partimos en este trabajo, sin embargo en el diseño de algunas pruebas -Emparejamiento, Verificación, Analogias-se han seleccionado sólo 4 de ellas por la complejidad que implicaria el manipular todas ellas.
} 
5. Relaciones semánticas:
A. Funcional
B. Parte-todo
C. Evaluativo o perceptual
D. Taxonómica.

6. Dificultades de los items
A. Baja
B. Alta

Resumiendo, los cuatro factores citados son factores o variables categoriales, pues afectan a la selección de los items, pero en el diseño de la Batería, como ya se ha dicho, también se han tenido en cuenta factores que tienen que ver con las pruebas o tareas: modalidad de presentación (verbal/oral versus pictórica/visual) y procesos cognitivos implicados en la resolución de dichas tareas (producción versus comprensión).

\section{COMENTARIOS ESPECIFICOS DE ALGUNAS DE LAS PRUEBAS}

En este apartado se comentarán solamente aspectos de algunas de las ocho pruebas, en concreto de cuatro de ellas: dos de producción y dos de comprensión, que son aquéllas a partir de las cuales se ha generado mayor número de trabajos y profundización en determinados aspectos de la Batería.

\section{Prueba 1: Fluidez verbal}

Aunque en su momento se consideró una prueba que evaluaba exclusivamente memoria semántica, hoy día se ha profundizado lo suficiente en el análisis de la tarea de producción de palabras de categorías, o tarea de fluidez verbal -en la secuenciación de las categorías producidas, en los clústers resultantes, etc. - como para haber podido constatar que, además de evaluar la integridad del conocimiento semántico y el acceso al léxico, esta prueba evalúa aspectos de la función ejecutiva, por lo cual muchos autores la sitúan en este contexto y no en el semántico estrictamente (Peña-Casanova, 2000).

A partir de los datos obtenidos en esta prueba de Fluidez Verbal de Categorias Semánticas (que parte de cinco categorías), se han publicado unas Normas de Producción, que se han completado con datos de una muestra de jóvenes equiparada a los ancianos sanos en determinadas variables demográficas de interés: género y nivel educativo (Peraita, Galeote, Díaz y Moreno, 2001). Son probablemente las primeras normas en español que presentan datos de jóvenes, de ancianos sanos y enfermos de Alzheimer para 5 categorías semánticas, consideradas cada una de ellas por separado y también conjuntamente, $\mathrm{y}$ recogiendo también los errores de producción para cada categoría y tipo de muestra.

\section{Prueba 2: Definición de categorias}

Especial interés merece la prueba de Definición de categorías, así como su complementaria, Reconocimiento de atributos, que no están diseñadas de manera ateórica -como sí lo han estado la mayoría de los análisis de atributos o propiedades en los trabajos de estos últimos años hasta el 2000 (con algunas excepciones como McRae y Cree (2002), Cree y McRae (2003), Wu y Barsalou (2002) y Simmons y Barsalou (2003) y pocos más) -y de los que hay muy pocos ejemplos en nuestro pais-, sino que parte de un modelo o esquema organizativo (Peraita, Elosúa y Linares, 1992; Peraita, Linares y Elosúa, 1990), que es un esquema de organización conceptual de categorias semánticas, válido tanto para las categorías de SVs como para las de SNVs.

Esta prueba consta de seis categorias de nivel supraordenado (cinco idénticas a la de la prueba uno, Fluidez Verbal) y sus correspondientes de nivel básico, es decir, evalúa el conocimiento de un total de doce categorias semánticas por medio de las definiciones verbales de dichas categorfas dadas por los sujetos en un tiempo determinado (ver Tabla 1).

Además de los trabajos publicados en los años 90 arriba citados, realizados a partir de los datos de producción de atributos en muestras de niños y adolescentes, algunos bilingües, y de todos los niveles de escolaridad, así como de muestras especiales (niños ciegos de nacimiento), se han publicado en esta línea de análisis de atributos de categorías otros dos trabajos a partir de los datos obtenidos por medio de la batería EMSDA en enfermos de Alzheimer y controles sanos (Peraita, Galeote y González Labra, 1999; Peraita y Moreno, 2005).

En los primeros (Peraita, Elosúa y Linares, 1992; Peraita, Linares y Elosúa, 1990) ya avanzamos (incluso bastante antes de trabajos muy 


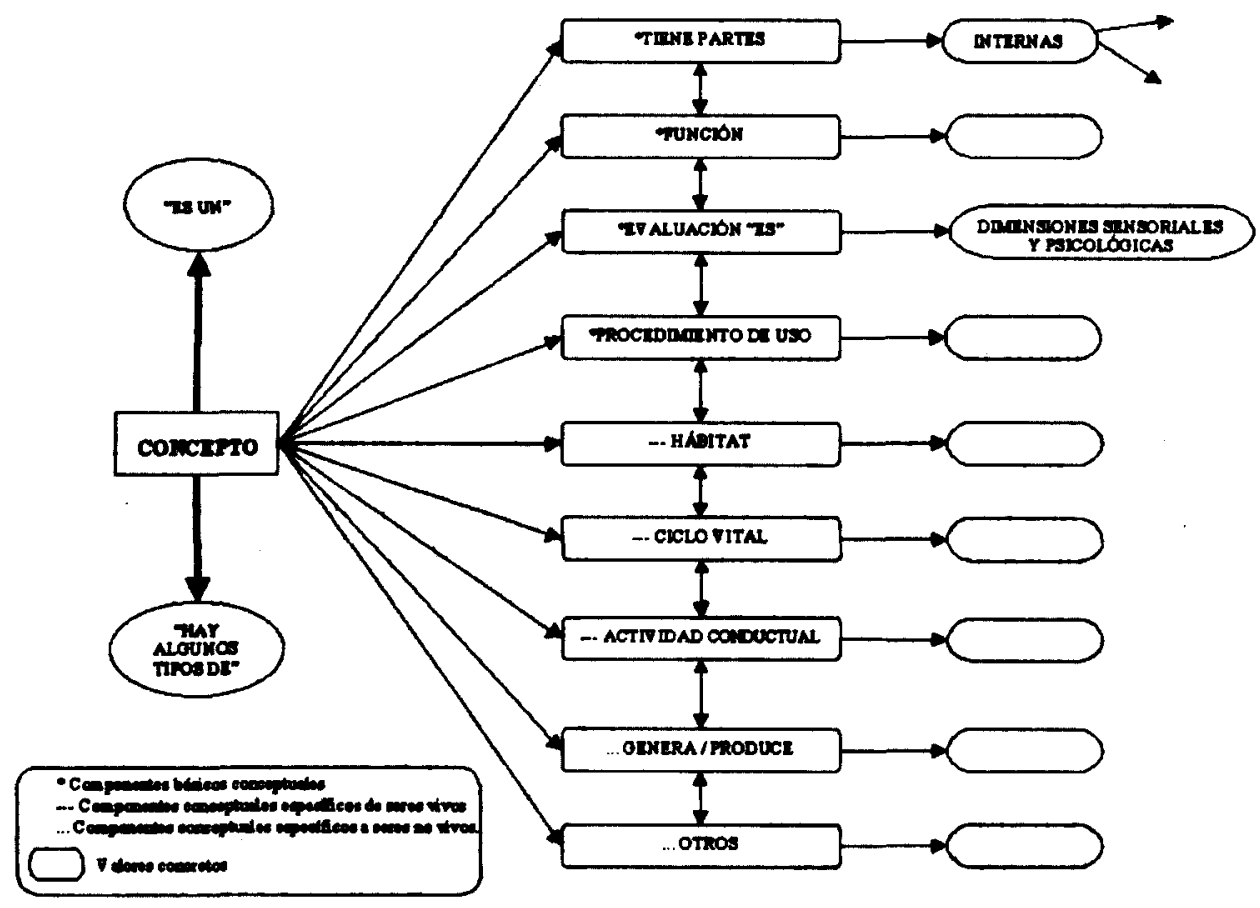

Figura 1. Modelo de representación de categorias semánticas de seres vivos y no vivos basado en rasgos o atributos (adaptado de Peraita, Elosúa y Linares, 1992).

recientes, como Simmons y Barsalou; 2003), la importancia del trabajo de Damasio y colaboradores $(1989 ; 1990)$ basado en el concepto de zonas de convergencia en la teorización sobre la estructuración y organización de los atributos de categorias, y tratamos de establecer un paralelismo entre los componentes conceptuales de nuestro modelo y los citados por Damasio (Peraita y Dobato, 1998) (ver Figura 1).

\section{Prueba 5: Emparejamiento palabra oida-dibujo}

De esta prueba interesa destacar el diseño de la estructura de los distractores, a través del cual se pretende obtener información sobre algunas de las hipótesis vigentes en este campo, a saber, integridad o deterioro de los dos grandes dominios semánticos - SVs y SNVs- y evaluación de dos de las relaciones semánticas mas importantes, la relación parte-todo (Peraita y Malrieu, 1998) y la penceptual-visual, a partir de los patrones de error. De la importancia de los atributos perceptuales visuales da una idea el hecho de que trabajos muy recientes los abordan explícitamente (Schweizer y Dixon, 2006; Mondini, Borgo, Cotticelli y Bisiacchi, 2006). (Ver Figura 2, a y b).

\section{Prueba 6: Verificación de enunciados}

Es una prueba de juicios semánticos sobre categorías 0 , lo que es lo mismo, de verificación de la capacidad del sujeto para evaluar la verdad o falsedad de atributos de categorías semánticas presentados en forma de enunciados. Consta de cuarenta y ocho enunciados, divididos en tres series de dieciséis items cada una. En cada una de estas series la mitad de los items se refiere a seres vivos y la otra mitad a seres no vivos y además la mitad son verdaderos y la mitad son falsos. Las dos primeras tienen el mismo nivel de dificultad, mientras que la tercera es de mayor dificultad que las anteriores. Esta prueba nos permite discernir lo que son simplemente problemas de acceso al léxico de lo que son verdaderas dificultades semán- 


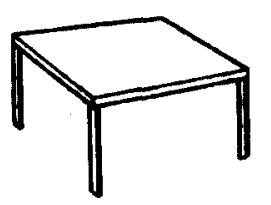

a)

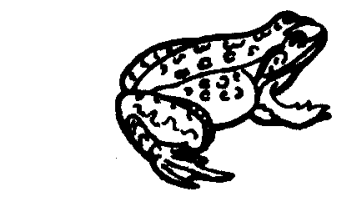

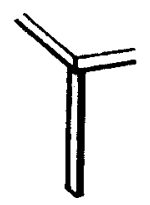

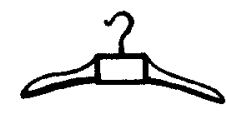

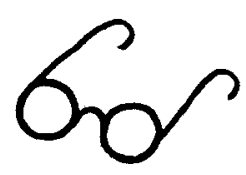

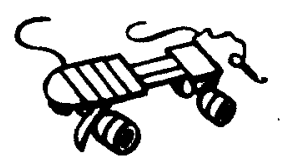

b)
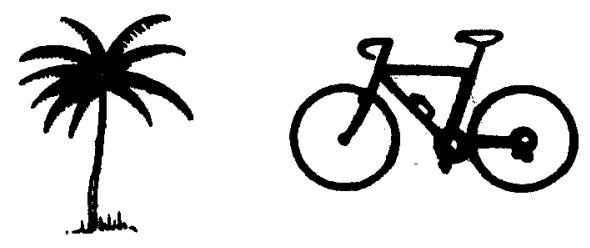

Figura 2 (a y b). Ejemplos de la prueba de Emparejamiento

ticas, como han indicado recientemente Mondini y colaboradores (2006) en una tarea de diseño prácticamente idéntico.

Los resultados de esta prueba son especialmente interesantes por la dificultad de los enfermos de Alzheimer para evaluar las relaciones semánticas falsas, no así las verdaderas. Dichos resultados se resumieron en Diaz, Peraita y Moreno (2001) y, más recientemente, se han analizado e interpretado con mayor detalle (Peraita, Díaz y Anllo-Vento, 2006). Nuestro equipo utilizó los resultados de este estudio como punto de partida en un estudio electrofisiológico reciente sobre priming semántico, cuyo objetivo fue determinar si el tipo de relación semántica entre dos palabras modificaba el tipo de procesamiento y la red cerebral involucrados en el mismo (Anllo-Vento et. al., 2004).

\section{NIVELES DE DIFICULTAD}

En tres de las ocho pruebas, Emparejamiento, Verificación y Analogias, se establecieron dos series de distinto nivel de dificultad, en función de la tipicidad de los items, justamente porque se habia comprobado en un estudio piloto que, en los participantes sanos, se producía el efecto techo. En estas pruebas los participantes realizaban la segunda serie sólo si hablan superado con éxito la primera -es decir, si hablan cometido menos de dos errores en la primera serie-.

De la misma manera, en la prueba de Clasificación se diseñaron dos modalidades: clasificación libre y semidirigida. Sin embargo, la enorme dificultad que suponía para los enfermos de Alzheimer la realización de la primera de estas modalidades, ha aconsejado actualmente retirar dicha prueba de la batería.

Además de los niveles de dificultad manipulados por nosotros en algunas de las tareas, algunas de las ocho de que consta la Baterla EMSDA son inherentemente más dificiles que otras y presentan, por tanto, mayores obstáculos de procesamiento cognitivo. En concreto, son de mucha mayor dificultad todas aquellas pruebas en las que, además de tener que acceder al conocimiento semántico almacenado (almacén semántico), se requiere recuperación de piezas léxicas y, por tanto, una respuesta explícita de tipo lingüístico. En concreto éstas últimas son: Fluidez Verbal, Definición de Categorias y Reconocimiento de Atributos, mientras que las imponen una menor carga cognitiva serian: Emparejamiento, Verificación, Clasificación Semidirigida y Analogias. Cabe señalar, sin embargo, que el componente inferencial de las analogias introdujo una dificultad adicional en esta prueba. (Ver Figura 3, a y b).

\section{TRABAJOS ANTERIORES EN QUE SE BASÓ EL DISEÑO DE LA BATERÍA EMSDA}

El diseño de algunas de las pruebas, como ya se ha hecho constar en la de Definición de Categorias, procede de trabajos anteriores de los colaboradores de la bateria EMSDA. En concreto, la de Empare- 

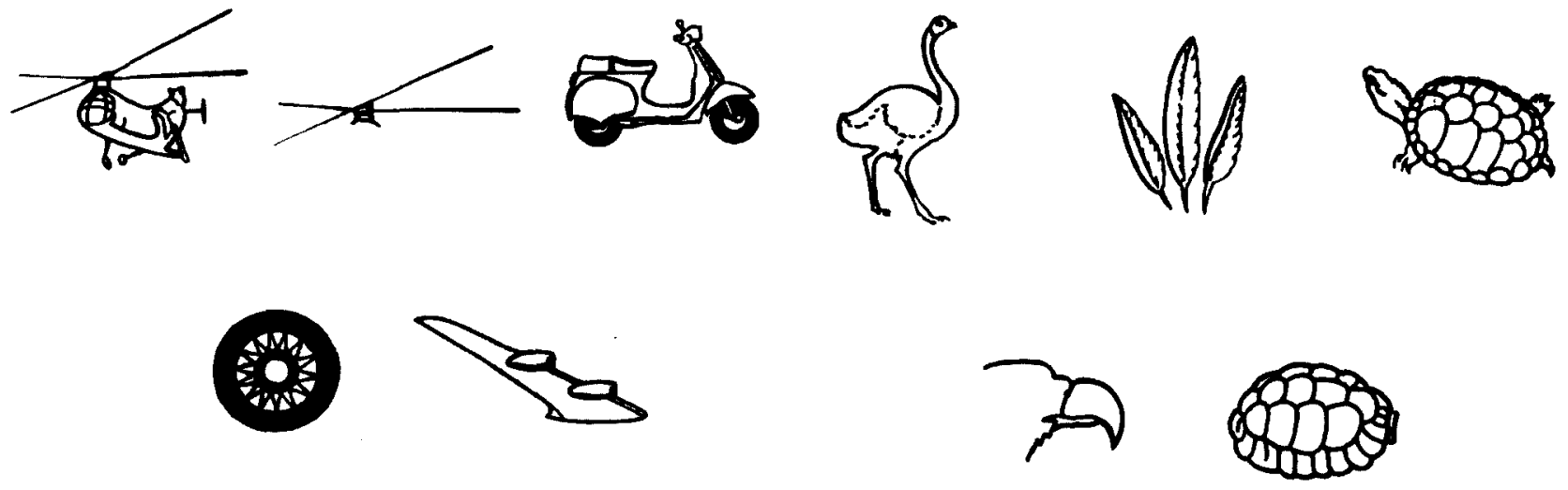

a)

b)

Figura 3 (a y b). Ejemplos de la prueba de Analogías semánticas

Tabla 2. Especificación del tipo de items de cada una de las pruebas en función de su pertenencia a las categorías de SVs y SNVs y niveles de dificultad de las mismas

\begin{tabular}{|c|c|c|c|c|c|c|c|}
\hline \multirow{3}{*}{$\begin{array}{l}\text { PRUEBAS } \\
I^{a} \text { Fluidez Verbal }\end{array}$} & \multirow[b]{2}{*}{ Nltems } & \multicolumn{6}{|c|}{ Items } \\
\hline & & \multicolumn{3}{|c|}{$S V s$} & \multicolumn{3}{|c|}{$S N V_{s}$} \\
\hline & 5 & \multicolumn{3}{|c|}{ Animales, plantas, frutas } & \multicolumn{3}{|c|}{ Prendas de vestir, vehículos } \\
\hline $2^{a}$ Definición & 12 & \multicolumn{3}{|c|}{ Animal, perro, árbol, pino, fruta, manzana } & \multicolumn{3}{|c|}{$\begin{array}{l}\text { Vehículo, coche, prendas de vestir, pantalón, } \\
\text { muebles, silla }\end{array}$} \\
\hline $3^{a}$ Denominación & 36 & $\begin{array}{l}\text { Animales } \\
\text { Perro } \\
\text { Canario } \\
\text { Lagarto } \\
\text { Sardina } \\
\text { Mosca } \\
\text { Mono }\end{array}$ & $\begin{array}{l}\text { Frutas } \\
\text { Manzana } \\
\text { Pera } \\
\text { Plátano } \\
\text { Coliflor } \\
\text { Lechuga } \\
\text { Alcachofa }\end{array}$ & $\begin{array}{l}\text { Plantas } \\
\text { Pino } \\
\text { Rosa } \\
\text { Abeto } \\
\text { Clavel } \\
\text { Cactus } \\
\text { Helecho }\end{array}$ & $\begin{array}{l}\text { Muebles } \\
\text { Silla } \\
\text { Mesa Cama } \\
\text { Sillón Armario } \\
\text { Sofá }\end{array}$ & $\begin{array}{l}\text { Vehiculos } \\
\text { Coche } \\
\text { Bicicleta } \\
\text { Avión } \\
\text { Moto } \\
\text { Camión } \\
\text { Autobús }\end{array}$ & $\begin{array}{l}\text { Prendas vestir } \\
\text { Pantalón } \\
\text { Camisa } \\
\text { Falda } \\
\text { Calcetines } \\
\text { Jersey } \\
\text { Chaqueta }\end{array}$ \\
\hline $4^{*}$ Reconocimiento & 12 & \multicolumn{3}{|c|}{ Animal, perro, árbol, pino, fruta, manzana } & \multicolumn{3}{|c|}{$\begin{array}{l}\text { Vehículo, coche, prendas de vestir, pantalón, } \\
\text { muebles, silla }\end{array}$} \\
\hline $5^{a}$ Emparejamiento & 28 & $\begin{array}{l}I^{\circ} \text { nivel } \\
\text { Perro, } \\
\text { pino, } \\
\text { plátano, st } \\
\text { rosa, } \\
\text { alcachofa, } \\
\text { cactus, }\end{array}$ & iente, & $\begin{array}{l}2^{\circ} \text { nivel } \\
\text { Campanillas, } \\
\text { foca, } \\
\text { coco, } \\
\text { sauce, } \\
\text { tortuga } \\
\text { canario }\end{array}$ & $\begin{array}{l}1^{\circ} \text { nivel } \\
\text { Camisa, } \\
\text { coche, } \\
\text { pantaló } \\
\text { mesa, } \\
\text { biciclets } \\
\text { avión, } \\
\text { armario } \\
\text { sofá }\end{array}$ & , calcetines, & $\begin{array}{l}2^{\circ} \text { nivel } \\
\text { Carretilla, } \\
\text { mecedora, } \\
\text { cinturón, carroza }\end{array}$ \\
\hline
\end{tabular}


Tabla 3. Especificación del tipo de items de cada una de las pruebas en función de su pertenencia a las categorías de SVs y SNVs y niveles de dificultad de las mismas (continuación)

\begin{tabular}{|c|c|c|c|c|c|c|c|}
\hline \multirow[t]{2}{*}{$\begin{array}{l}\text { 6“ Verificación } \\
7^{a} \text { Clasificación }\end{array}$} & \multirow[t]{2}{*}{$\begin{array}{l}48 \\
\\
18\end{array}$} & \multirow{2}{*}{\begin{tabular}{l}
\multicolumn{1}{c}{$I^{a}$} \\
Manzana, \\
pino \\
mosca, \\
mar, \\
sardina, \\
paloma, \\
pajaro \\
Animales \\
Foca, \\
lechuza, \\
tortuga
\end{tabular}} & \multirow{2}{*}{\multicolumn{2}{|c|}{\begin{tabular}{l}
\multicolumn{1}{c}{$2^{a}$} \\
Sauce, \\
foca \\
fresón, \\
lechuza, \\
campanillas \\
\\
$\begin{array}{l}\text { Plantas } \\
\text { Sauce, } \\
\text { enredadera, } \\
\text { campanillas }\end{array}$
\end{tabular}}} & \multicolumn{2}{|c|}{\begin{tabular}{l}
\multicolumn{1}{c}{$l^{\star}$} \\
Coche, \\
sartén, \\
camisa, \\
pelota, \\
aguja, \\
armario, \\
silla
\end{tabular}} & \multirow{2}{*}{\begin{tabular}{c}
\multicolumn{1}{c}{$2^{a}$} \\
$\begin{array}{l}\text { Perchero, vestido, } \\
\text { linterna, carretilla; } \\
\text { brocha }\end{array}$ \\
$\begin{array}{l}\text { Prendas de vestir } \\
\text { Sostén, } \\
\text { chaqueta, } \\
\text { cinturón }\end{array}$
\end{tabular}} \\
\hline & & & & & $\begin{array}{l}\text { Muebles } \\
\text { Perchero, } \\
\text { mecedora, } \\
\text { litera }\end{array}$ & $\begin{array}{l}\text { Vehiculos } \\
\text { Carretilla, } \\
\text { barca, } \\
\text { carroza }\end{array}$ & \\
\hline $8^{a}$ Analogias & 18 & $\begin{array}{l}I^{\circ} \text { nivel } \\
\text { Buey, } \\
\text { lagarto, } \\
\text { canario, } \\
\text { rosa, } \\
\text { avestruz, 1 } \\
\text { manzana }\end{array}$ & $\begin{array}{r}\text { Ca } \\
\text { búl }\end{array}$ & $\begin{array}{l}2^{\circ} \text { nivel } \\
\text { panillas, fresón, }\end{array}$ & \multicolumn{2}{|c|}{$\begin{array}{l}\quad l^{\circ} \text { nivel } \\
\text { Perchero, coche, } \\
\text { helicóptero, zapato, } \\
\text { cama }\end{array}$} & $\begin{array}{l}2^{\circ} \text { nivel } \\
\text { Paquetes, } \\
\text { mecedora, } \\
\text { cómoda }\end{array}$ \\
\hline Total Items & 177 & & & & & & \\
\hline
\end{tabular}

jamiento, con su estructura de diferentes tipos de distractores (que tenia por finalidad originalmente analizar los patrones de error en afásicos), fue diseñada por Sánchez Bernardos (Sánchez Bernardos, 1988) y la prueba de Analogías por González Labra (González Labra, 1983), basándose en los trabajos de Vosniadou y Ortony (1989) y suponian una extensión de trabajos anteriores de González Labra sobre analogias geométricas. Esta prueba es de gran interés en la evaluación de la memoria semántica porque permite estudiar su deterioro mediante una tarea distinta a las tradicionalmente utilizadas, y no sólo evalúa los procesos de acceso y recuperación de la información sino su utilización en el establecimiento de nuevas relaciones semánticas (Ceacero y González Labra, 1998). La selección de los items estuvo de alguna manera determinada por resultados del trabajo de Galeote (1982).

\section{AMPLIACIÓN Y ACTUALIZACIÓN DE LA BATERIA EMSDA}

En el momento actual está llevándose a cabo la ampliación y extensión de algunos aspectos de dicha batería, así como la profundización consiguiente, a lo largo de los siguientes ejes:

1. Introducción de modalidades sensoriales distintas de la visión, pero relacionadas con otros canales sensoriales implicados también en la representación semántica tanto de los SVs como de los SNVs: olfativa/olor, acústica/sonido, somestésica/tacto, gustativa/sabor.

2. Profundización en la modalidad de interacción motora con los objetos para estudiar las praxias, por medio de verbos - ausentes de la primera versión de la Batería - y acciones motoras.

3. Réplica y/o profundización del trabajo de McRae y Cree (2002), para comprobar si los datos obtenidos en una lengua distinta del inglés -el español en nuestro caso- mantienen la misma estructura o soporte conceptual de atributos o propiedades semánticas, y eventual revisión del modelo descrito en Peraita y colaboradores (1992).

\section{REFERENCIAS}

Anllo-Vento, L., Hinojosa, J. A., Silva, A. I., Pozo, M. A., y Peraita, H. (2004). Modulation of the N400 by the 
type of semantic relationship connecting word pairs in a semantic priming task. Cognitive Neuroscience Society. San Francisco.

Ceacero, J., y González-Labra, M.J. (1998). El razonamiento analógico como solución de problemas. Introducción a la Psicologia del Pensamiento (pp. 409. 453). Madrid: Trotta.

Cree, G., y McRae, K. (2003). Analyzing the Factors Underlying the Structure and Computation of the Meaning of Chipmunk, Cherry, Chisel, Cheese, and Cello (and Many Other Such Concrete Nouns). Journal of Experimental Psychology, 132, 163-201.

Damasio, A.R. (1989). Time-locked multiregional retroactivation: A system-level proposal for the neural substrates of recall and recognition. Cognition, 33, 25-62

Damasio, A.R. (1990). Category-related recognition defects as a clue to the neural substrates of knowledge. Trends in Neuroscience, 13, 95-98.

Diaz, C. (2003). Seguimiento del deterioro de la memoria semántica de enfermos de Altheimer evahuados mediante la Bateria EMSDA. (Tesis doctoral no publicada. UNED).

Díaz, C., Peraita, H., y Moreno, F.J. (2001). Estudio comparativo de pruebas de denominación y emparejamiento palabra-dibujo en Enfermos de Alzheimer. Estudios de Psicologia, 22/3.

Galeote, M. (1982). La formación de categorias naturales: un estudio evolutivo y diferencial. Madrid: Universidad Complutense de Madrid.

González Labra, M.J. (1983). El razonamiento analógico: Variables de la tarea con figuras geométricas. Revista de Psicología General y Aplicada, 38, 927-946.

McRae, K., y Cree, G. (2002). Factors underlying category-specific semantic deficits. En E.M.E. Forde \& G. Humphreys (Eds.), Category-specificity in mind and brain. East Sussex, Uk: Psychology Press.

Mondini, S., Borgo, F., Cotticelli, B., y Bisiacchi, P. (2006). Progressive knowledge loss: A longitudinal case study. Journal of the International Neuropsychological Society, 12, 275-284.

Peña-Casanova, J. (2000). Programa integrado de exploración neuropsicológica. Test Barcelona (Manual). Barcelona: Masson, S.A.

Peraita, H., Diaz, C., y Anllo-Vento, L. Difficulty in processing various types of semantic relations in patients with Alzheimer's disease (En proceso de revision en Archives of Clinical Neuropsychology).

Peraita, H., y Dobato, J.L. (1998). Modeles neurophysiologiques sur la déterioration de la memoire conceptuelie chez les patients d'Alzheimer: confrontation de deux modbles. Journees de Rochebrune: Rencontres interdisciplinaires sur les systèmes complexes naturels et artificiels. Telecom Paris. ENST. $98 \mathrm{~S} 001$.

Peraita, H., Elosua, R., y Linares, P. (1992). Representación de categorias naturales en niños ciegos de nacimiento. Madrid: Trotta.

Peraita, H., Galeote, M., Díaz, C., y Moreno, J. (2002).
Normas de producción de categorias semánticas. Estudios de la UNED. Madrid: UNED.

Peraita, H., Galeote, M., y González-Labra, M.J. (1999). Deterioro de la memoria semántica en pacientes de Alzheimer: evidencia a partir de tareas de definición, clasificación y razonamiento analógico. Psicothema, 11, 917-937.

Peraita, H., González-Labra, M.J., Sánchez Bernardos, M. L., y Galeote, M. (2000). Batería de evaluación del deterioro de la memoria semántica en Alzheimer. Psicothema, 12, 192-200.

Peraita, H., González-Labra, M.J., Sánchez Bernardos, M. L., y Galeote, M. (2001). www.Psychologyinspain.com/content/full/2001/10.htm, 5, 1,98-109

Peraita, H., Linares, P., y Elosúa, R. (1990). Conceptual representation: Thoughts and suggestions from data on a sample of blind subjects. Actas Cognitiva, 90, 851-856.

Peraita, H., y Malrieu, D. (1998). La relevancia de las partes en el sistema léxico-conceptual humano. Estudios de Psicologia, 60, 111-128.

Peraita, H., y Moreno, F.J. Análisis de la estructura conceptual de las categorias semánticas naturales y artificiales en una muestra de pacientes de Alzheimer. Psicothema. (Aceptado en Diciembre 2005.Ref.2005/142).

Rosch, E. R., Mervis, C.B., Gray, W.D., Jonson, D.M., y Boyes-Braem, P. (1976). Basic objects in natural categories. Cognitive Psychology, 8, 382-439.

Sénchez Bernardos, M.L. (1988). La distinción forma-significado y su contribución al estudio de la afasia anómica. Tesis doctoral no publicada. Madrid: UCM.

Schweizer, T.A., y Dixon, M. J. (2006). The influence of visual and nonvisual attributes in visual object identification. Journal of the International Neuropsychological Society, 12, 176-183.

Simmons, W. K. y Barsalou, L.W. (2003). The similarity-intopography principle: Reconciling theories of conceptual deficits. Cognitive Newropsychology, 20, 451-486.

Smith, E.E., y Medin, D. L. (1981). Categories and concepts. Cambridge, M.A: Harvard University Press.

Smith, E.E., Shoben, E.J., y Rips, L. J. (1974). Structure and processes in semantic memory. A featural model for semantic decisions. Psychological Review, 81, 214-241.

Snodgrass, J.G., y Vanderwart, M. (1980). A standardized set of 260 pictures: Norms for name agreement, image agreement, familiarity and visual complexity. Journal of Experimental Psychology, 6, 174-208.

Soto, P, Scbastitin, M.V., Gancla, E., y del Amo, T. (1994). Las Categorias y sus normas en castellano. Madrid: Visor.

Vosniadou, S., y Ortony, A. (1989). Similarity and Analogical Reasoning. Cambridge: Cambridge University Press.

Wu, L., y Barsalou (2002): Grounding Concepts in Penceptual Simulation: 1. Evidence from Property Genenation.

Herminia Peraita

Universidad Nacional de Educación a Distancia, Madrid 